\title{
VIEWING ANGLES IN SOME SELECTED BL Lac OBJECTS
}

\author{
F.J. ZHANG \\ Shanghai Observatory, Chinese Academy of Sciences, Shanghai 200030, China \\ L.B. BÅÅTH \\ Center for Imaging Technologies, Halmstad University, Sweden
}

(Received 26 April, 1995; accepted 28 September 1995)

\begin{abstract}
A selected subset of BL Lac objects were monitored with VLBI techniques at multifrequencies. Apparent superluminal motion and X-rays were detected in all of the monitored sources. From the observational data, kinematic parameters, geometric parameters and beaming effects for the 'core' of the selected BL Lac objects have been estimated. Our results indicate that some BL Lacs have large Lorentz factors and small viewing angles; relativistic beaming effects are strongly present in these sources. On the other hand, some BL Lacs have small Lorentz factors; their viewing angles are quite large. It does not seem possible to avoid the fact that some BL Lac objects are not oriented particularly close to the line of sight. It conflicts with the basic assumption of the accepted theories for explaining the characteristics of BL Lac objects at present. Also, it seems that the BL Lac objects in the class I of the Burdige and Hewitt's classification have larger viewing angles than those int he class II. The whole BL Lac classification could be a misnomer; the sources classified as 'BL Lac objects' are a mixture of physically different populations with different parent objects.
\end{abstract}

\section{Introduction}

It is unclear What kind of sources BL Lac objects are. Usually, to be called a BL Lac object the source must show rapid variability and/or high linear optical polarization, and a featureless or nearly featureless optical spectrum. They have unusual statistical properties compared with other types of high-luminosity active galactic nuclei (AGN). The generally accepted explanation is that the observed continuum has been amplified relative to the other components. BL Lac objects were originally proposed to be the extreme end of the quasar population with jets seen end-on $[8,9]$. Synchrotron radiation from the core in BL Lac objects overwhelms all the other components.

There are indications that a single class of "BL Lac objects" does not exist. Burbidge and Hewitt [12] have divided BL Lacs into two classes: "BL Lacs in galaxies", mainly nearby sources with $z<0.3$, and "BL Lacs with QSO spectra", all with $z>0.3$. Recently, they have suggested that the sources in the second category should be reclassified as QSOs [13]. A similar classification has been suggested by Browne [10]. He divided BL Lac objects into two classes: class a, those which lie in the centres of elliptical galaxies; and class b, those objects with broad lines, which almost certainly form a subclass of quasars. The whole BL Lac classification could be a misnomer: the sources classified as 'BL Lac objects' are a mixture of physically different populations with different parent objects. 